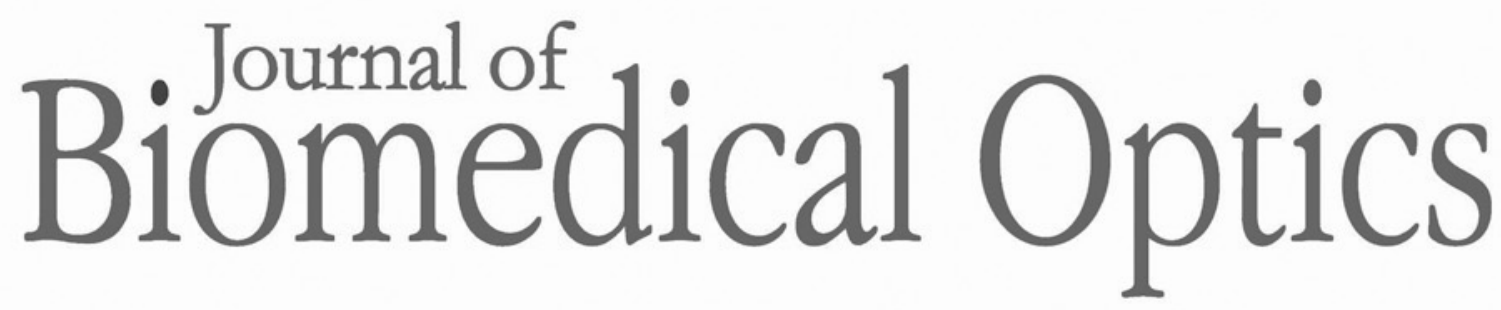

\title{
Three-dimensional quantitative phase imaging of blood coagulation structures by optical projection tomography in flow cytometry using digital holographic microscopy
}

\author{
Hideki Funamizu \\ Yoshihisa Aizu
}




\title{
Three-dimensional quantitative phase imaging of blood coagulation structures by optical projection tomography in flow cytometry using digital holographic microscopy
}

\author{
Hideki Funamizu* and Yoshihisa Aizu \\ Division of Production Systems Engineering, Muroran Institute of Technology, Muroran, Japan
}

\begin{abstract}
Blood coagulation is an important role in the hemostasis process. In the observation using microscopies, an aggregation structure of red blood cells indicates the degree of blood coagulation. Recently, it has been proposed that digital holographic microscopy (DHM) is a powerful tool for biomedical cell imaging on the basis of quantitative phase information. DHM has the advantage in that the real-time and three-dimensional (3-D) quantitative phase imaging can be realized in the wide field of view, which means that the 3-D morphological parameters of biological cells without a staining process are obtained in real time. We report the complete 3-D quantitative phase imaging of blood coagulation structures by optical projection tomography in a flow cytometry using DHM. @ The Authors. Published by SPIE under a Creative Commons Attribution 4.0 Unported License. Distribution or reproduction of this work in whole or in part requires full attribution of the original publication, including its DOI. [DOI: 10.1117/1.JBO.24.3.031012]
\end{abstract}

Keywords: digital holographic microscopy; red blood cell; blood coagulation; optical projection tomography; quantitative phase imaging; flow cytometry.

Paper 180402SSR received Jul. 2, 2018; accepted for publication Sep. 20, 2018; published online Oct. 9, 2018.

\section{Introduction}

It is known that blood coagulation is an important role in hemostasis related to the cessation of blood loss from a damaged blood vessel. Coagulation disorders increase a risk of hemorrhage or thrombosis, and lead to severe diseases in blood circulation. Many different techniques have been proposed and developed to detect the degree of blood coagulation or to observe an aggregation structure of red blood cells (RBCs). ${ }^{1-10}$ An observation of RBCs and blood coagulation structures using microscopic techniques is useful for biomedical imaging of cell morphology, and therefore cell imaging methods in various types of microscopies are proposed, such as electron microscopy,${ }^{7}$ atomic force microscopy, ${ }^{8}$ confocal optical microscopy, ${ }^{9}$ and conventional optical microscopy. ${ }^{10}$ Although electron and atomic force microscopies realize the 3-D imaging with a high spatial resolution, these microscopies are invasive to biological cells, have the small field of view, and need to perform a scanning process with time consumption, which means that it is difficult to realize the real-time measurements in these microscopies. A confocal microscopy can observe biological cells noninvasively, but it has the same disadvantages in the field of view and in the acquisition time of the 3-D information as the electron and atomic force microscopies. A conventional optical microscopy can noninvasively observe biological cells in the wide field of view in comparison with other microscopies, while the scanning process for the 3-D imaging is required in the depth direction. The scanning process significantly reduces the time resolution of microscopic systems, which means that it is difficult to realize the real time and 3-D measurement in these microscopies. Therefore, the realization of such types of

*Address all correspondence to: Hideki Funamizu, E-mail: funamizu@mmm .muroran-it.ac.jp measurement methods opens new possibilities in the area of biological imaging, such as the 3-D measurements of living and moving cells in real time.

Recently, it has been proposed that digital holographic microscopy (DHM) is a powerful tool for biomedical cell imaging, and it is actively applied to the blood testing on the basis of quantitative phase information. ${ }^{11-15}$ DHM has the advantage in that a thickness of biological cells, which is regarded as the depth information in an observation of cell morphology using quantitative phase microscopies in many cases, is obtained from a single hologram and therefore the real time and 3-D quantitative phase measurement can be realized in the wide field of view. It means that the 3-D morphological parameters and activities of biological cells without a staining process can be obtained using DHM in real time. Therefore, DHM plays an important role in the medical and biological areas of cell imaging, such as the measurement of thickness, a refractive index, and cell morphology of biological cells, ${ }^{16,17} 3$-D cell tracking, ${ }^{18-23}$ cell counting, ${ }^{24,25}$ cell recognition and identification on the basis of morphological parameters of cells, ${ }^{26-30}$ and so on.

In a conventional DHM, as a laser light is vertically incident on biological cells, an optical field generated from them is projected in a propagation direction of the laser light, and then the projected optical field is recorded on an image sensor as a digital hologram. In the reconstruction process, the projected phase map of the optical field is reconstructed from the digital hologram and has phase values integrated over the optical path of the laser light. Therefore, while the thickness of cells can be obtained from the projected phase map, the complete 3-D structures cannot be reproduced from it due to the integral of the phase modulation in the optical path. In addition, as a quantitative phase value of biological cells in DHM is derived as the multiplication of the refractive index and thickness of cells, it is 
assumed that either of the two parameters is known in many cases. Although it is often assumed that the refractive index is known in the case of RBCs because they have a homogeneous refractive index distribution, this assumption causes large measurement errors in the observation of biological cells with an inhomogeneous refractive index distribution. One of the methods for the complete reproduction of the 3-D structures and distributions of refractive indices is the 3-D tomographic imaging in quantitative phase microscopy, for which several methods have been proposed using various quantitative phase microscopies. $^{31-36}$

In this study, we propose a quantitative phase imaging of blood coagulation structures using DHM and report the 3-D tomographic imaging of aggregation structures of RBCs formed by the blood coagulation process using quantitative phase distributions in DHM. The 3-D tomographic phase imaging of blood coagulation structures is performed by optical projection tomography in a flow cytometry using DHM. To our knowledge, this paper is the first report of the 3-D tomographic phase imaging of blood coagulation structures using DHM.

\section{Optical Setup}

Figure 1 shows the optical system in this study. We adapt the Mach-Zehnder DHM in an off-axis configuration. A laser diode (Mitsubishi ML101J27, $660.0 \mathrm{~nm}, 120 \mathrm{~mW}$ ) is used as the optical source. The laser light is divided by the half mirror $\mathrm{HM}$. One of the split lights is reflected by a mirror $\mathrm{M}_{3}$ and is cleaned by an objective lens $\mathrm{OB}_{2}$ and a spatial filter $\mathrm{SF}$. It is then collimated by a lens $\mathrm{L}$ and is used as a reference wave. The light transmitted through $\mathrm{HM}$ is reflected by $\mathrm{M}_{4}$ and is incident on an object, which is a flow cell in Fig. 1. This wave is used as an object wave. It is magnified by $\mathrm{OB}_{3}(\times 40, \mathrm{NA}=0.65)$ and is collimated by a tube lens TL with a focal length of $150 \mathrm{~mm}$. In this setup, an afocal configuration is applied to DHM and the magnification of it is 34.1 times power. The object and reference waves are coupled by a beam splitter BS again, and then these waves interfere each other. The interference pattern, which is called a hologram, is detected on a charge-coupled device (CCD) camera (AVT, GC2450) with $2448 \times 2050$ pixels, a pixel pitch $3.45 \mu \mathrm{m}$, and a frame rate $15 \mathrm{fps}$. The CCD camera is located at the back focal plane of TL and therefore image



Fig. 1 Experimental setup of a Mach-Zehnder DHM in an off-axis setup. $\mathrm{LD}$, laser diode; $\mathrm{OB}$, objective lens; $\mathrm{M}$, mirror; $\mathrm{HM}$, half mirror; L, lens; SF, spatial filter; and BS, beam splitter.

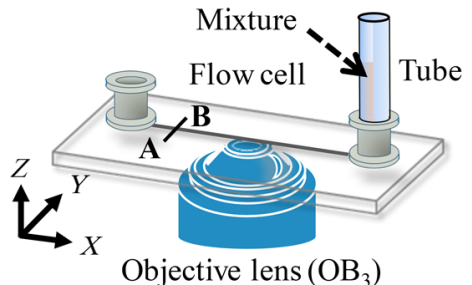

Fig. 2 Schematic of the sample.

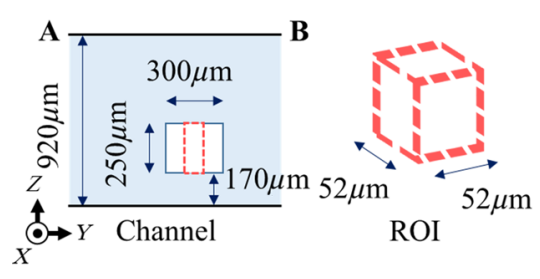

Fig. 3 Details of the flow cell and ROI in DHM.

holograms are recorded. To separate DC terms and a twin image from an object image in the reconstruction plane, holograms are modulated by a spatial carrier, which is added by adjusting the angle of BS suitably. Holograms are saved into computer and are reconstructed by numerical calculation using the spatial filtering method. ${ }^{37}$

Figures 2 and 3 show a schematic diagram of a flow cell and the region of interest in DHM. The sample is the mixture of horse blood (Nippon Bio-test Laboratory Inc.) and the coagulation time reagent (Sysmex, BR002733). The hematocrit value of horse blood is $25 \%$, which is measured by the experiment using a centrifugal separator and hematocrit capillary tube. After that, the horse blood is adjusted to the hematocrit $0.1 \%$ with plasma having the refractive index of 1.332. The coagulant solution is produced by dissolving the coagulation time reagent in $4 \mathrm{~mL}$ of purified water with the refractive index of 1.333 . After warming both liquids at $37.0^{\circ} \mathrm{C}$ for $30 \mathrm{~min}$, we mix $0.1 \mathrm{~mL}$ of the horse blood and $0.2 \mathrm{~mL}$ of the coagulant solution. The refractive index of the mixture surrounding the RBCs is $n_{s}=1.333$, because the refractive indices of the plasma and the purified water are almost the same values and the coagulant solution is mixed more than the horse blood. The mixture is withdrawn into one side of a tube by a syringe, and another side of the tube is connected to the flow cell (Translume Inc., SF-300250-170-L) composed of the fused silica with the refractive index of 1.456 . The mixture is injected by pushing the piston of the syringe by the automicrometer. While the blood coagulation structures are fixed by fibrin networks, they are broken with an increase in the flow speed. Therefore, the flow speed is adjusted to keep the blood coagulation structures by the automicrometer, suitably. The focus of DHM is adjusted in the bottom of the channel ( $300 \mu \mathrm{m}$ in width, $250 \mu \mathrm{m}$ in depth). In this setup, a laser light is vertically incident on the flow cell, and RBCs flow while rotating in the direction from one side of the flow path to the other in the flow cell as shown in Fig. 4. The movie of digital holograms of the rotating RBCs in the flow cell, from which the projected phase maps at any angles are reconstructed, is recorded on the CCD camera. To increase the number of the samples of digital holograms in the rotation angle, the image size of the CCD camera is reduced to $512 \times 512$ pixels, and therefore the frame rate increases to 


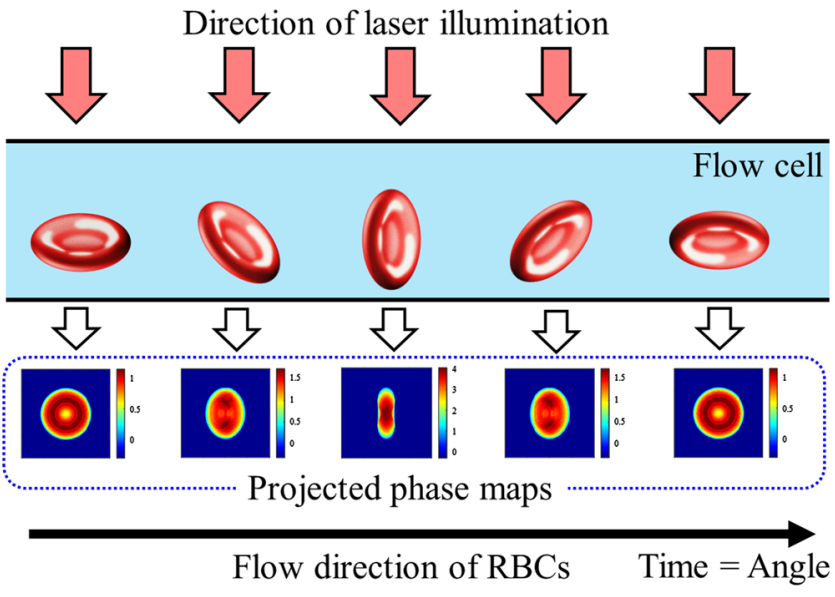

Fig. 4 Schematic of the rotation of RBCs and the acquisition of projected phase maps.

39.1 fps. Under these conditions, the field of view of the reconstructed images is $52 \times 52 \mu \mathrm{m}^{2}$.

\section{Optical Projection Tomography Using Phase Maps Reconstructed from Digital Holograms}

Figure 5 shows the analytical process of optical projection tomography in flow cytometry using DHM. ${ }^{36,38}$ The 3-D structures of RBCs are reproduced by applying optical projection tomography to projected phase maps reconstructed from the movie of digital holograms. In the reconstruction process, phase distributions are reconstructed from digital holograms using the spatial filtering method. After that, a phase unwrapping process is applied to the phase distributions, in which the fast-Fourier-based phase unwrapping is used. ${ }^{39}$ This process needs not to perform a single RBC, but it is necessary for the blood coagulation structures because the phase values of them are wrapped at several rotation angles. As RBCs flow in the flow cell, the position of RBCs is different in each hologram. To adjust the positions of RBCs for each hologram and apply optical projection tomography to projected phase maps, the center of gravity of RBCs is calculated on the basis of phase information reconstructed from each hologram. First, a phase map of RBCs is trimmed by the square area with $128 \times$ 128 pixels for a single $\mathrm{RBC}$ and $256 \times 256$ pixels for blood coagulation structures. Next, the binalization process is applied to the trimmed image, and then the filling process is performed to remove holes in the binary image. After these processes, the center of gravity of RBCs is estimated from the binary image of RBCs. For the RBCs in the reconstructed image of the next flame, the same processes are applied, except that the phase map of the RBCs is trimmed using the center of gravity of the RBCs of the previous flame as the center of the trimming region. Under these experimental conditions in the present study, the phase distributions of RBCs of the next flame can be successfully trimmed and traced using this method without fail. These processes are applied to all flames of the phase maps in the reconstructed images. After matching the position of RBCs using the center of gravity, optical projection tomography is performed to the projected phase maps.

In optical projection tomography of RBCs, we use the filtered back projection (FBP) method. In this study, the maximum angle for the sinogram is estimated from the phase information of RBCs by the cross-correlation method and is set to $\pi \mathrm{rad}$. Therefore, the angle for the sinogram is in the range from 0 to $\pi \mathrm{rad}$. We estimate the RBCs of a flame, which is rotated by $\pi \mathrm{rad}$ for them of the first flame. When the cross-correlation coefficient of phase maps of RBCs in the first flame and other flames is $>0.97$, the rotating angle of the RBCs is regarded as $\pi$ $\mathrm{rad}$, in which the phase images of other flames are mirrorreversed image due to the rotation of RBCs. The angle separation is determined by dividing the maximum of the rotation angle by the flame number from the first flame to the estimated flame. Therefore, the angle of rotation of RBCs linearly corresponds to time in the movie of holograms. After performing these processes in the rotation angle, row vectors of projected phase maps are aligned on the time axis, which is equal to the angle axis with each angle separation, to produce sinogram of the row. By applying the FBP method to the sinogram, the 2-D tomographic image of the phase distribution of RBCs is produced. In this method, the product of the Fourier transform of each projection and the Shepp-Logan filter is



Fig. 5 Analytical process of optical projection tomography in flow cytometry using DHM. 
inverse-Fourier transformed, and then the 2-D tomographic image is reconstructed by performing the back projection of all filtered data. After applying these processes to all rows of phase maps, the 3-D tomographic data of RBCs is reproduced by stacking the 2-D tomographic images. The tomographic phase data are converted into the refractive index distribution $n_{R}(X, Y, Z)$ of RBCs in the $3-\mathrm{D}$, which is given as

$n_{R}(X, Y, Z)=\frac{\lambda}{2 \pi \Delta z} \Phi(X, Y, Z)+n_{s}$,

where $\lambda$ is the wavelength of the optical source, $\Phi(X, Y, Z)$ is the phase value of the 3-D tomographic data, and $\Delta z$ is the pixel size of the 3-D tomographic data in the $z$-direction and is the same as the pixel size of the reconstructed image in the $X$ - and $Y$-directions.

\section{Results and Discussion}

Figures 6(a)-6(c), 6(d)-6(f), and 6(g)-6(i) show the results of a single RBC and the blood coagulation structures composed of three and nine RBCs, respectively. The left column in Fig. 6 is the phase maps reconstructed from a single digital hologram at a rotation angle. The center column in Fig. 6 shows the volume slice plane of the 3-D tomographic data of the refractive index distributions. The right column in Fig. 6 is the 3-D outer shapes of RBCs, which are obtained by applying the binalization process to the 3-D tomographic data of the refractive index distributions shown in the center column of Fig. 6. In these images, we use the refractive index 1.370 as the threshold of the binalization process. In the process of optical projection tomography, the number of holograms, which corresponds to the number of projected phase maps, is 92,46 , and 51 for a single RBC and the blood coagulation structures composed of three and nine RBCs, respectively. It is seen from these figures that the 3-D tomographic image of blood coagulation structures is successfully reproduced from projected phase maps reconstructed from digital holograms. In the refractive index distributions shown in Figs. 6(b), 6(e), and 6(h), the average value of the refractive index is 1.41 , which agrees well with the literature value. ${ }^{30}$ It is found in Figs. 6(c), 6(f), and 6(i) that the complete

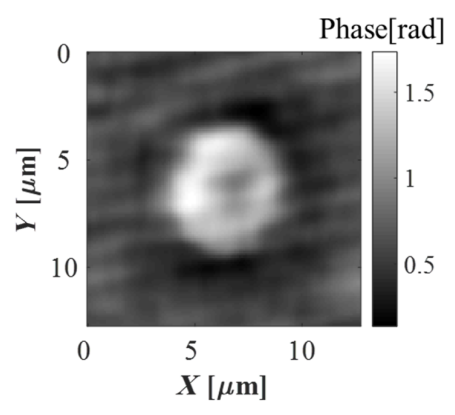

(a)

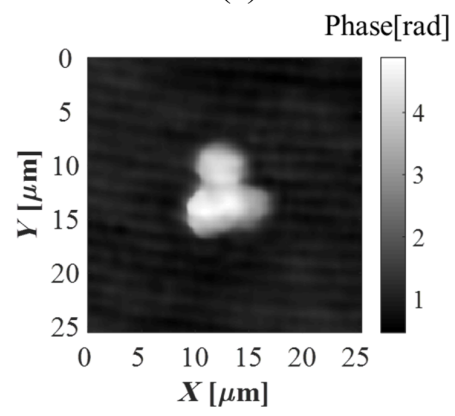

(d)



(g)

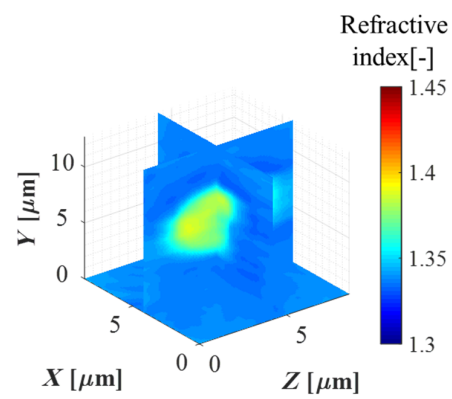

(b)

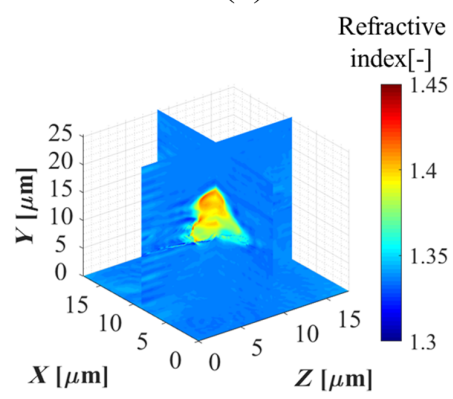

(e)

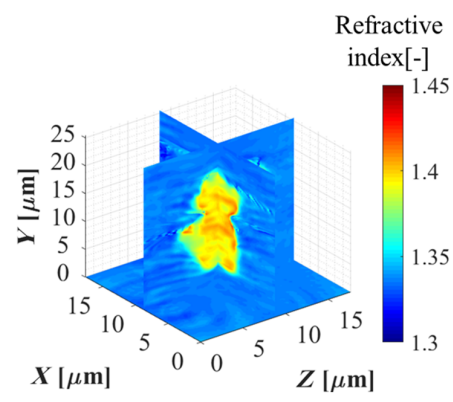

(h)

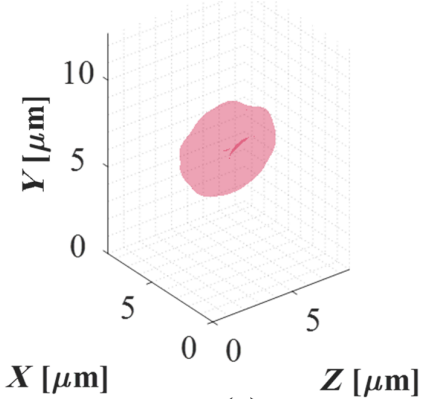

(c)



(f)

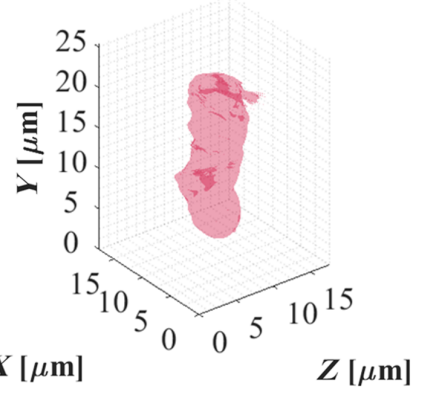

(i)

Fig. 6 Experimental results of optical projection tomography in the flow cytometry using DHM. (a)-(c), (d)-(f), and (g)-(i) show the results of a single RBC and the blood coagulation structures of three and nine RBCs, respectively. (a), (d), and (g) are the phase maps reconstructed from holograms at a rotation angle. (b), (e), and (h) are the results of the volumetric slice plane of the 3-D tomographic image of the refractive index distributions. (c), (f), and (i) show the 3-D outer shapes of RBCs. 
3-D structures are recovered using the tomographic imaging on the basis of quantitative phase data and these involve important features in comparison with a conventional DHM. In a single $\mathrm{RBC}$, while the tomographic imaging technique is useful for the complete recovery of the 3-D structure, it may be sufficient to be characterized by a single projected phase map and various geometrical parameters obtained from it. On the other hand, as the blood coagulation structures have more complex 3-D structures than a single RBC, it is insufficient to characterize them from a single projected phase map, and therefore it is more important for the blood coagulation structures to recover the complete 3-D structures and to characterize their structures using the 3-D geometrical parameters on the basis of the 3-D outer shape shown in Figs. 6(c), 6(f) and 6(i).

However, it is seen from Figs. 6(e) and 6(h) that the fluctuation of the refractive index distributions occurs in the region without RBCs, which implies that the noise level of Figs. 6(e) and 6(h) is higher than that of Fig. 6(b). This is because the number of projected phase maps in optical projection tomography is smaller in the case of Figs. 6(e) and 6(h). The fluctuation of the refractive index distribution effects on the results in Figs. 6(f) and 6(i) as these results are produced by applying the binalization process to the $3-\mathrm{D}$ distributions of the refractive index.

\section{Conclusion}

In this study, we reported the 3-D tomographic imaging of aggregation structures of RBCs formed by the blood coagulation process using quantitative phase distributions in DHM. For the 3-D tomographic imaging of blood coagulation structures, optical projection tomography was performed in a flow cytometry using DHM. In the experimental setup, we used the MachZehnder DHM of the off-axis and afocal configuration in the recording process and the spatial filtering method in the reconstruction process. The laser light was vertically incident on the flow cell, in which RBCs flow while rotating in the direction from one side of the flow path to the other in the flow cell. We acquired the digital holograms of rotating RBCs in the flow cell. Using projected phase maps reconstructed from digital holograms recorded at any angles of the rotating RBCs, optical projection tomography was performed for the blood coagulation structures, and the complete 3-D structures and refractive index distributions were successfully reproduced. To our knowledge, this paper is the first report of the tomographic imaging of blood coagulation structures using DHM.

Though several tomographic methods using quantitative phase imaging are proposed, the present method has several advantages in that the blood testing with the evaluation of blood coagulation structures on the basis of the complete 3-D structures of RBCs can be realized by means of the low-cost and portable quantitative phase microscopy with a small number of optical devices and simple optical systems, and therefore leads to the possibility of the realization of the point-of-care diagnosis systems of the blood test and the blood coagulation test using the complete 3-D structures recovered from tomographic phase imaging. In conclusion, it is believed that the results in this study have potential applications of blood coagulation testing in microscopic observations and analyses of various kinds of cells using quantitative phase microscopy, and will contribute to various medical and biological researches related to RBCs and blood coagulation, such as the understanding the formation mechanism of blood coagulation structures, early diagnosis and detection of the serious diseases related to blood coagulation phenomenon, the drug developments of these diseases, and so on.

\section{Disclosures}

No conflicts of interest, financial or otherwise, are declared by the authors.

\section{Acknowledgments}

This work was supported by JSPS KAKENHI Grant No. JP15K06098. We declare that no conflicts of interest exist for either author.

\section{References}

1. T. M. Wilson, "The conductivity of blood in coagulation," Biochem. J. 2, 377-382 (1907).

2. S. Kishimoto and M. Yoshioko, "Method and system for measuring blood coagulation time," U. S. Patent No. 4,252,536 (1981).

3. B. Kloth and H. Behnk, "Blood coagulation time measuring device," U. S. Patent No. 4,964,728 (1990).

4. F. A. Greco, "Reflectance spectroscopy of clotting blood: a description of the time-dependent behavior," Arch. Pathol. Lab. Med. 128(2), 173180 (2004).

5. M. Faivre et al., "Coagulation dynamics of a blood sample by multiple scattering analysis," J. Biomed. Opt. 16(5), 057001 (2011).

6. M. M. Tripathi et al., "Assessing blood coagulation status with laser speckle rheology," Biomed. Opt. Express 5, 817-831 (2014).

7. S. N. Mardel et al., "Reduced quality of clot formation with gelatinbased plasma substitutes," Br. J. Anaesth. 80, 204-207 (1998).

8. N. Yeow, R. F. Tabor, and G. Garnier, "Atomic force microscopy: from red blood cells to immunohaematology," Adv. Colloid Interface Sci. 249, 149-162 (2017).

9. A. Elnager et al., "In vitro whole blood clot lysis for fibrinolytic activity study using d-dimer and confocal microscopy," Adv. Hematol. (2014).

10. V. Chaikeeratisak, A. Tassanakajon, and P. B. Armstrong, "Interaction of pathogenic vibrio bacteria with the blood clot of the pacific white shrimp, litopenaeus vannamei," Biol. Bull. 226(2), 102-110 (2014).

11. M. K. Kim, Digital Holographic Microscopy, Springer, New York (2011).

12. T. Zhang and I. Yamaguchi, "Three-dimensional microscopy with phase-shifting digital holography," Opt. Lett. 23(15), 1221-1223 (1998).

13. E. Cuche, F. Bevilacqua, and C. Depeursinge, "Digital holography for quantitative phase-contrast imaging," Opt. Lett. 24(5), 291-293 (1999).

14. J. Garcia-Sucerquia et al., "Digital in-line holographic microscopy," Appl. Opt. 45, 836-850 (2006).

15. J. Kühn et al., "Real-time dual-wavelength digital holographic microscopy with a single hologram acquisition," Opt. Express 15(12), 72317242 (2007).

16. C. J. Mann et al., "High-resolution quantitative phase-contrast microscopy by digital holography," Opt. Express 13, 8693-8698 (2005).

17. A. Anand, V. K. Chhaniwal, and B. Javidi, "Imaging embryonic stem cell dynamics using quantitative 3-d digital holographic microscopy," IEEE Photonics J. 3, 546-554 (2011).

18. W. Xu et al., "Tracking particles in four dimensions with in-line holographic microscopy," Opt. Lett. 28, 164-166 (2003).

19. I. Bernhardt et al., "Application of digital holographic microscopy to investigate the sedimentation of intact red blood cells and their interaction with artificial surfaces," Bioelectrochemistry 73(2), 92-96 (2008).

20. Y.-S. Choi and S.-J. Lee, "Three-dimensional volumetric measurement of red blood cell motion using digital holographic microscopy," Appl. Opt. 48, 2983-2990 (2009).

21. P. Langehanenberg et al., "Automated three-dimensional tracking of living cells by digital holographic microscopy," J. Biomed. Opt. 14(1), 014018 (2009).

22. X. Yu et al., "Four-dimensional motility tracking of biological cells by digital holographic microscopy," J. Biomed. Opt. 19(4), 045001 (2014).

23. X. Yu et al., "Review of digital holographic microscopy for threedimensional profiling and tracking," Opt. Eng. 53(11), 112306 (2014). 
24. S. Seo et al., "High-throughput lens-free blood analysis on a chip," Anal. Chem. 82(11), 4621-4627 (2010).

25. F. Yi, I. Moon, and Y. H. Lee, "Three-dimensional counting of morphologically normal human red blood cells via digital holographic microscopy," J. Biomed. Opt. 20(1), 016005 (2015).

26. B. Javidi, M. Daneshpanah, and I. Moon, "Three-dimensional holographic imaging for identification of biological micro/nanoorganisms," IEEE Photonics J. 2, 256-259 (2010).

27. M. Mihailescu et al., "Automated imaging, identification, and counting of similar cells from digital hologram reconstructions," Appl. Opt. 50, 3589-3597 (2011).

28. M. Mir, K. Tangella, and G. Popescu, "Blood testing at the single cell level using quantitative phase and amplitude microscopy," Biomed. Opt. Express 2, 3259-3266 (2011).

29. I. Moon et al., "Automated statistical quantification of three-dimensional morphology and mean corpuscular hemoglobin of multiple red blood cells," Opt. Express 20, 10295-10309 (2012).

30. P. Marquet, C. Depeursinge, and P. J. Magistretti, "Review of quantitative phase-digital holographic microscopy: promising novel imaging technique to resolve neuronal network activity and identify cellular biomarkers of psychiatric disorders," Neurophotonics 1(2), 020901 (2014).

31. F. Charrière et al., "Cell refractive index tomography by digital holographic microscopy," Opt. Lett. 31, 178-180 (2006).

32. W. Choi et al., "Tomographic phase microscopy," Nat. Methods 4, $717-719$ (2007)

33. K. Kim et al., "Real-time visualization of 3-D dynamic microscopic objects using optical diffraction tomography," Opt. Express 21, 32269-32278 (2013).

34. A. Kuś et al., "Tomographic phase microscopy of living three-dimensional cell cultures," J. Biomed. Opt. 19(4), 046009 (2014).
35. M. Habaza et al., "Tomographic phase microscopy with $180^{\circ}$ rotation of live cells in suspension by holographic optical tweezers," Opt. Lett. 40, 1881-1884 (2015).

36. F. Merola et al., "Tomographic flow cytometry by digital holography," Light: Sci. Appl. 6, e16241 (2017).

37. E. Cuche, P. Marquet, and C. Depeursinge, "Spatial filtering for zeroorder and twin-image elimination in digital off-axis holography," Appl. Opt. 39(23), 4070-4075 (2000).

38. J. Sharpe et al., "Optical projection tomography as a tool for $3 \mathrm{~d}$ microscopy and gene expression studies," Science 296(5567), 541-545 (2002).

39. S. V. D. Jeught, J. Sijbers, and J. J. J. Dirckx, "Fast Fourier-based phase unwrapping on the graphics processing unit in real-time imaging applications," J. Imaging 1(1), 31-44 (2015).

Hideki Funamizu is an associate professor at Muroran Institute of Technology, Japan. He received his PhD in electronics and information engineering from Hokkai-Gakuen University in 2008, Japan. From 2008 to 2010, he was an assistant professor at Tokyo University of Science, Japan. In 2010, he joined Muroran Institute of Technology, Japan. His current research activities are in the biomedical optical imaging, digital holographic technique, and speckle measurements.

Yoshihisa Aizu received his Dr Eng from Hokkaido University in 1985. In 1989, he joined Hokkaido University as a research associate. From 1990, he was an associate professor at Muroran Institute of Technology, and in 2006 he has been promoted a professor, the position he now holds. From 1992 to 1993, he was a visiting researcher in University Erlangen, Germany. His research activities are in biomedical applications of spectroscopy and optical imaging. 